\title{
Constructal law optimization of a boiler
}

\author{
Teresa M. Gulotta ${ }^{1 *}$, Francesco Guarino ${ }^{1}$, Maurizio Cellura ${ }^{1}$, Giulio Lorenzini ${ }^{2}$ \\ ${ }^{1}$ Department of Energy, Information Engineering and Mathematical Models, Viale delle Scienze \\ Building. 9, Università degli Studi di Palermo, Palermo, 90128, Italy \\ ${ }^{2}$ Department of Engineering and Architecture, Parco Area delle Scienze no. 181/A Università \\ degli Studi di Parma, 43124 Parma, Italy
}

Email: teresamaria.gulotta@unipa.it

\begin{abstract}
The paper aims at the optimization of the design of a biomass boiler under the inspiration of the Constructal Law by Bejan. The boiler is of the smoke tubes typology, fuel being biomass pellets. The smoke tubes are 16 and are placed in a staggered configuration. A model is built in MATLAB environment, based on empirical correlations and the mean log temperature methodology. The analysis is based on the development of a wide parametric analysis that involves variations of diameters, numbers and positioning of the tubes. Results are based on the concept of the overall performance coefficient methodology and investigate both the pressure drops variation and the thermal power generated in the different configurations' boiler. However, since the aim of the boiler is to guarantee a fixed thermal power output to the users, results are also presented by fixing the thermal power output and adding as variable the volume of tubes adopted as an indicator to quantify materials required to achieve the same output. While from the point of view of pressure losses there is a clear best solution, this is not the case if the material uses minimization and pressure drops minimization are jointly analyzed. This leads to the need to a trade-off in performance to identify a best case.
\end{abstract}

Keywords: Boiler, Constructal Law, Modelling, Parametric Analysis, Overall Performance Coefficient.

\section{INTRODUCTION}

The "Constructal theory", proposed in 1997 by Bejan is based on a simple principle: for fluids, the best geometry is the one that exerts the lesser resistance to motion; instead for the transfer of thermal energy, the best geometry is the one that generates the least thermal resistance[1]. Constructal theory is the view that the generation of flow configuration is a universal phenomenon of all physics, which is covered by a law of physics (the Constructal law):

"For a finite-size flow system to persist in time (to live) it must evolve such that it provides greater and greater access to the currents that flow through it" [2].

Also, Bejan explains the connection between "Constructal law" and the thermodynamics of flow systems by developing analytical and graphical formulations to maximize flow access in systems with heat and fluid flow irreversibility and freedom to change configuration [3].

Constructal theory has been applied to various fields, from heat and mass transfer applications in engineered systems to biology, geophysics, social dynamics and economics fields.

In particular, there are several research works of Constructal Law on heat exchangers [4-10] and on the optimal shaping of fins with application to heat exchangers [11].
For example, the objective of [12] is the design of a heat exchanger that takes maximum advantage of high heat transfer density achieved by the use of small-scale channels with laminar flow. It is shown that the spacing of the elemental and first-construct channels can be optimized such that the overall pumping power required is minimal.

In [13], a Constructal Law analysis was conducted on a blast furnace iron-making process. The process is optimized and the results show that the hot metal yield and useful energy are, respectively, increased by $3.13 \%$ and $2.66 \%$ after generalized Constructal optimization.

In [14] the problem of determining the configuration of a stream that must be heated in a fixed space while using minimal fuel is proposed, applied to the heating of a stream of solid metal in a furnace. The results show that the distribution of heaters per unit area depends on the shape of the floor and the density of heaters per unit area varies as

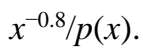

In other cases, it has been shown how to increase the power generation over the area occupied by the solar chimney configuration[15]. In this case it was found that the power generated per unit of land area is proportional to the length scale of the power plant.

Although energy applications of the Constructal Law are widespread in literature, there is only a limited availability of 
studies on the specific topic of optimizing the thermal performances of a boiler.

The investigation performed is based on an optimization of the design of a biomass boiler through the application of the Constructal Law.

The work approaches also the perspective of eco-design, including the use of materials required to achieve a fixed heating power as parameter of the optimization.

In particular the aim of the work is to apply the Constructal Law and the adaptive overall performance coefficient developed by Lorenzini et al. [16] to perform an optimization of the geometrical design of a pellet boiler through a performance index dependent on the overall pressure drop and the quantity of building materials.

\section{METHODS}

The following paragraphs discuss the technical characteristics of the actual boiler, the mathematical model implemented and the methodology chosen for the study.

\subsection{The boiler}

The analysis is based on the study of an existing boiler. Its most relevant technical and geometrical data are reported in Table 1. Figure 1 shows a picture of the boiler.

Table 1. Geometrical and technical data of the boiler

\begin{tabular}{lll}
\hline Characteristics & Unit & Value \\
\hline Diameter & $\mathrm{mm}$ & 50 \\
\hline Length & $\mathrm{m}$ & 0.6 \\
\hline Width & $\mathrm{m}$ & 0.6 \\
\hline Height & $\mathrm{m}$ & 0.6 \\
\hline Number of tubes & - & 16 \\
\hline Flow rate smoke tubes & $\mathrm{kg} / \mathrm{s}$ & 0.829 \\
\hline Flow rate water & $\mathrm{kg} / \mathrm{s}$ & 0.245 \\
\hline Thermal power & $\mathrm{kW}$ & 46 \\
\hline Efficiency & - & $89 \%$ \\
\hline
\end{tabular}

The boiler is of the smoke tubes typology, fuel being biomass pellets. The smoke tubes are 16 and are placed in a staggered configuration.

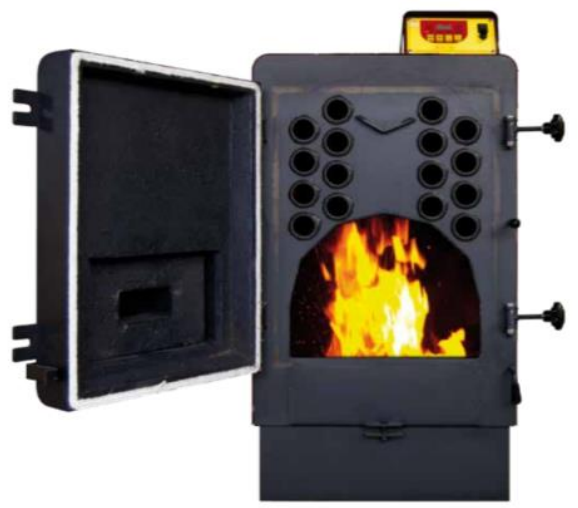

Figure 1. Photo of the boiler analyzed
Biomass pellets are burnt in the combustion chamber, hot smokes enter the tubes visible in Figure 1 and leave from a chimney placed on the back of the boiler. Water exchanges heat with the smoke tubes entering from the left in cross flow configuration.

\subsection{Mathematical modeling}

To analyze the existing system, a stationary mathematical model was developed in MATLAB environment, implementing empirical correlations and the method of the log-mean temperature difference to determinate the heat transfer between flows.

The model can undergo change of the boundary conditions, geometrical configuration of the heat exchanger, variations of the physical - chemical characteristics of the fluids, while respecting the laws of the thermo-fluid dynamics. It can be applied to any kind of similar boilers of the same kind using the same working principles.

The model implements heat transfer in the flow across a bank of tubes through the energy balance equation for a cross flow heat exchanger (Eq. 1) [17].

$$
\dot{Q}=\left(\dot{m} c_{p}\right)_{t}\left(T_{t, i}-T_{t, o}\right)=\left(\dot{m} c_{p}\right)_{s}\left(T_{s, o}-T_{s, i}\right)
$$

where $\mathrm{Q}$ is the heat duty in $\mathrm{kW}, \dot{m}$ is the mass flow rate $[\mathrm{kg} / \mathrm{s}], \mathrm{c}_{\mathrm{p}}$ is the specific heat capacity $\left[\mathrm{kJ}(\mathrm{kgK})^{-1}\right], \mathrm{T}$ is the temperature $[\mathrm{K}]$, the subscripts $\mathrm{t}$ and $\mathrm{s}$ stand for the tube-side and shell-side, respectively; and the subscripts $\mathrm{i}$ and o stand for the inlet and outlet of tube or shell side, respectively.

Moreover, the heat transferred in the heat exchanger can be calculated from the following equation 2 .

$\dot{Q}=F U A \lambda T_{L M}$

where $\mathrm{F}, \mathrm{U}, \Delta T_{L M}$ and $\mathrm{A}$ are respectively a correction factor that it is a function of the geometrical arrangement and of the temperatures of both flow, heat transfer coefficient $\left[\mathrm{W}\left(\mathrm{m}^{2}\right.\right.$ $\left.\mathrm{K})^{-1}\right]$, the logarithmic mean temperature difference between the two flows $[\mathrm{K}][18,19]$ and the heat transfer area $\left[\mathrm{m}^{2}\right]$.

Eq. 3 shows the calculation of logarithmic mean temperature difference.

$\Delta \mathrm{T}_{\mathrm{LM}}=\frac{\left(\mathrm{T}_{\mathrm{s}, \mathrm{i}}-\mathrm{T}_{\mathrm{t}, \mathrm{o}}\right)-\left(\mathrm{T}_{\mathrm{s}, \mathrm{o}}-\mathrm{T}_{\mathrm{t}, \mathrm{i}}\right)}{\ln \left(\left(\mathrm{T}_{\mathrm{s}, \mathrm{i}}-\mathrm{T}_{\mathrm{t}, \mathrm{o}}\right) /\left(\mathrm{T}_{\mathrm{s}, \mathrm{o}}-\mathrm{T}_{\mathrm{t}, \mathrm{i}}\right)\right)}$

The value of heat transfer area (A) is calculated in Eq.4.

$A=\pi \cdot d_{o} \cdot L \cdot N$

where $L$ is length of tubes $[\mathrm{m}], \mathrm{d}_{\mathrm{o}}[\mathrm{m}]$ is outside diameter of the tube and $\mathrm{N}$ is the total tube number

The shell volumetric hydraulic diameter $\left(D_{h}\right)$ is calculated as follow [19]:

$D_{h}=\frac{4 A_{0} W}{A}$

where $A_{0}$ is the passage area $\left[\mathrm{m}^{2}\right]$ of the cross-flow to bank of tubes and it is calculated in Eq. 6 and $\mathrm{W}$ is the width of boiler $[\mathrm{m}]$. 
$A_{o}=\left(P_{T}-d_{o}\right) \cdot L \cdot N_{r}$

where $\mathrm{N}$ is the tube number, $\mathrm{d}_{\mathrm{o}}[\mathrm{m}]$ is outside diameter of the tube, $S_{T}[\mathrm{~m}]$ and $S_{L}[\mathrm{~m}]$ are, respectively, the transversal and longitudinal pitch and $\mathrm{P}_{\mathrm{T}}$ and $\mathrm{P}_{\mathrm{L}}$ are the dimensionless pitch, calculated as ratio between pitch and outside diameter and the subscript $r$ stand for the rows of tubes in longitudinal direction.

The thickness of the pipes is equal to $5 \mathrm{~mm}$, as in the real case.

The thermal transmittance (U) is calculated using Eq. 7 and it is expressed in $\mathrm{W} /\left(\mathrm{m}^{2} \mathrm{~K}\right)$.

$\frac{1}{U}=\frac{1}{h_{o}}+\mathrm{R}_{\mathrm{f}, \mathrm{o}}^{\prime \prime}+\frac{\mathrm{d}_{\mathrm{o}} \cdot \ln \left(\mathrm{d}_{\mathrm{o}} / \mathrm{d}_{\mathrm{i}}\right)}{2 \mathrm{k}}+\mathrm{R}_{\mathrm{f}, \mathrm{i}}^{\prime \prime} \frac{\mathrm{d}_{o}}{\mathrm{~d}_{\mathrm{i}}}+\frac{1}{h_{i}} \frac{\mathrm{d}_{\mathrm{o}}}{\mathrm{d}_{\mathrm{i}}}$

where $R$ " is the fouling factor $\left[\left(\mathrm{m}^{2} \mathrm{~K}\right) / \mathrm{W}\right]$, and $\mathrm{h}\left[\mathrm{W}\left(\mathrm{m}^{2} \mathrm{~K}\right)\right.$ $\left.{ }^{1}\right]$ is the convection heat transfer coefficient and it is calculated as Eq. 8 .

$h=\frac{N u \cdot k}{D_{h}}$

where $\mathrm{Nu}$ is the Nusselt number, $\mathrm{k}[\mathrm{W} /(\mathrm{m} \mathrm{K})]$ is the conduction heat transfer coefficient and $D_{h}[\mathrm{~m}]$ is the hydraulic diameter.

This model calculates the Nusselt number and the pressure drop for bank of tubes as in the model of Zhukauskas as in [19] (Eqs. 9 and 10 respectively):

$N u_{\text {cold }}=C_{1} C_{2} \operatorname{Re}_{D, \max }^{m} \operatorname{Pr}^{n}\left(\frac{\operatorname{Pr}}{\operatorname{Pr}_{w}}\right)^{1 / 4}$

$\Delta P_{\text {cold }}=\chi N_{r}\left(\rho \frac{u_{\mathrm{max}, \text { cold }}^{2}}{2}\right) f_{\text {cold }}$

where $\mathrm{C}_{1}, \mathrm{C}_{2}, \mathrm{~m}, \mathrm{n}$, are dimensionless parameters that change in function of physical and geometrical characteristic of cross flow heat exchanger arrangement. Correction factor $C_{1}$ varies as function of Reynolds ( $\mathrm{Re})$ and arrangement of tubes. Instead, $f$ and $\chi$ are the friction factor and its correction factor $[18,19]$. If the number of rows of tubes is lower than 16 , it is necessary to use the correction factor $\mathrm{C}_{2}$.

Pressure drops internal to the tubes are calculated as Eq. 11.

$\Delta P_{h o t}=f_{h o t} \frac{u_{h o t}^{2}}{2} \frac{L}{d_{i}}$

where $f$ is the friction factor function of Re; $u$ is the speed $\left[\mathrm{ms}^{-1}\right] ; \mathrm{L}[\mathrm{m}]$ is length of tubes and $\mathrm{d}_{\mathrm{i}}$ is the inner diameter of tubes.

The hot smokes stream inlet temperature $\left(\mathrm{T}_{\text {hot,in }}\right)$ and the cold stream inlet temperature $\left(\mathrm{T}_{\text {cold,in }}\right)$ are fixed, since no modification is suggested to the combustion chamber and no modification in the water supply is expected during the use phase.

Mass flow rate is specified at the inlets of the hot and the cold stream. The fluid velocity is calculated as in Eqs. 12 and 13: $u_{h o t}=\frac{\dot{m}_{h o t}}{\rho_{h o t} A_{t}}$

$u_{\text {cold }}=\frac{\dot{m}_{\text {cold }}}{\rho_{\text {cold }} A_{\text {shell }}}$

where $u$ is the velocity of the flow $\left[\mathrm{ms}^{-1}\right]$ and $\rho$ is the density of the flow $\left[\mathrm{kg} \mathrm{m}^{-3}\right]$.

The Reynolds number $\mathrm{Re}_{\mathrm{D}, \max }$ for the foregoing correlation is based on the maximum fluid velocity, calculated in Eq. 14, occurring within the tube bank in Eq. 15.

$\operatorname{Re}_{h o t}=\frac{(\rho u)_{h o t} d_{i}}{\mu}$

$\operatorname{Re}_{D, \text { max }, \text { cold }}=\frac{\rho_{\text {cold }} u_{\text {max }} d_{o}}{\mu}$

For the fluid in the shell side, the maximum reachable speed depends on its geometrical shape configuration. The maximum speed is calculated through Eq. 16.

$u_{\max }=\left\{\begin{array}{cc}\frac{S_{T}}{S_{T}-d_{o}} u_{\text {cold }} & \text { for } S_{D} \geq \frac{S_{T}+d_{o}}{2} \\ \frac{S_{T}}{2\left(S_{D}-d_{o}\right)} u_{\text {cold }} & \text { for } S_{D}<\frac{S_{T}+d_{o}}{2}\end{array}\right.$

Eq. 17 is the diagonal distance between the centers of adjacent tubes, also called diagonal pitch $\left(\mathrm{S}_{\mathrm{D}}\right)$.

$S_{D} \equiv \sqrt{S_{L}^{2}+\left(\frac{S_{T}}{2}\right)^{2}}$

where $S_{T}[m]$ and $S_{L}[m]$ are, respectively, the transversal and longitudinal pitch.

\subsection{Parametric analysis}

The objective for the case studies is the minimization of the global pressure drop and the minimization of volume of materials in the boiler through a Constructal Law approach.

To determine the optimal Constructal Law design of the boiler, a parametric analysis was performed on the main geometrical parameters of the boiler.

In particular:

1. The tubes layout was varied into: staggered arrangement (as in the existing design, in Figure 2) or aligned arrangement (as in Figure 3);

2. The number of tubes $(\mathrm{N})$ is varied between five discrete values: $8,12,16,20$ and 24

3. The diameter of the tubes $(50 \mathrm{~mm})$ is varied between 30 and $150 \mathrm{~mm}$

The boiler has been designed with uniform element tube spacing and straight tubes as shown.

The geometrical models for the heat exchanger used can be referred to two different arrangements of the bank tubes. A tube bank usually consists of many rows of tubes both parallel and perpendicular to the flow direction. The tubes may be arranged in staggered or aligned configurations as 
shown in (

Figure 2) and (Figure 3), respectively.

The boiler control volume is shown in

Figure 2 and Figure 3 including also geometrical nomenclature (the length $\mathrm{L}$, the height $\mathrm{H}$ and the width $\mathrm{W}$ ).

The aligned configuration was modeled by integrating Eq.18 in substitution of Eq. 16 for the staggered configuration.

$u_{\max }=\frac{S_{T}}{S_{T}-d_{o}} u_{\text {cold }}$

Similar formulations of the dimensionless parameters $\left(C_{1}\right.$, $\mathrm{C}_{2}, \mathrm{~m}, \mathrm{n}, f$ and $\left.\chi\right)$ are implemented for the aligned configuration in [19].

The thickness of the pipes is considered constant for all simulation, indicated with $\mathrm{t}_{\text {wall }}$, equal to $2,5 \mathrm{~mm}$, as in the real case.

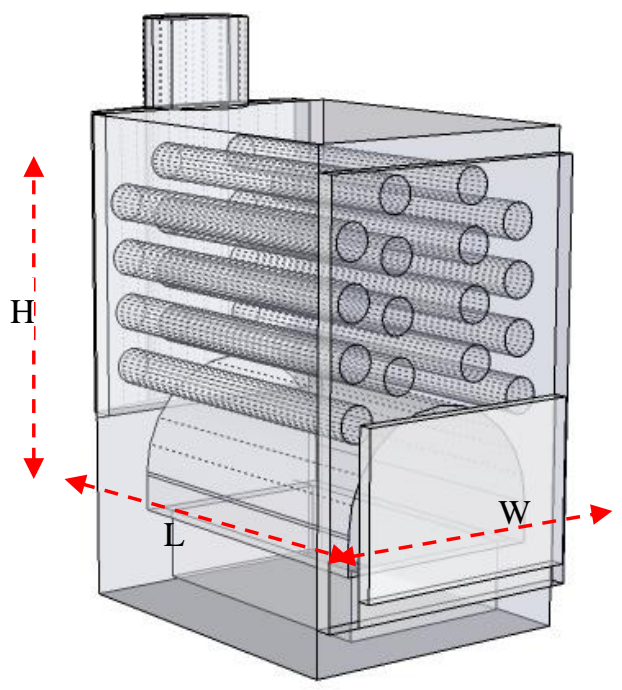

Figure 2. Basic mathematical model of boiler (real case)

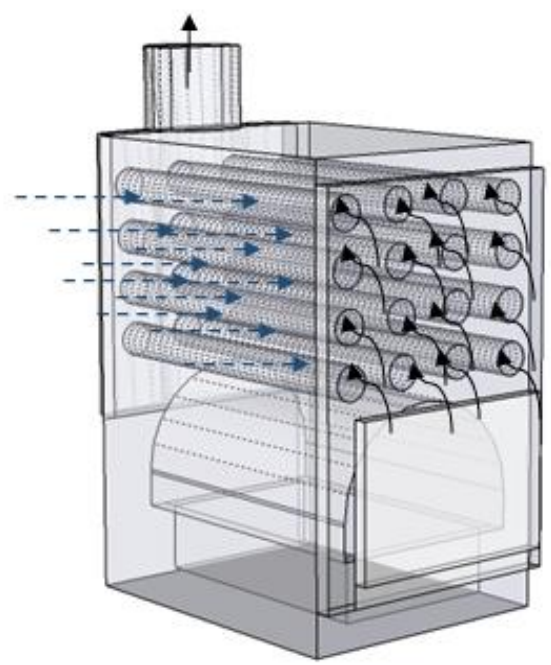

Figure 3. Basic mathematical model of boiler (Case 7)

Initially ten cases were defined, characterized by increasing values of number of tubes and different geometric arrangements. Table 2 resumes the parametric features of 10 cases, including the existing case.
These cases have been chosen to not alter the boundary characteristics of the original boiler, in fact, the height, the depth and the width do not vary throughout the parametric analysis.

Applying a variation to the number of tubes without changing the size of the boiler, a maximum variation in the diameter of the tubes was fixed so as to allow a section of sufficient passage of water equal to $10 \%$ of the distance between the centers of the tubes, as described in [18].

Table 2. Design parameters

\begin{tabular}{ccc}
\hline Case study & Geometrical configuration & Number of tubes \\
\hline Real case & Staggered & 16 \\
\hline$\# 1$ & Staggered & 8 \\
\hline$\# 2$ & Staggered & 12 \\
\hline$\# 3$ & Staggered & 20 \\
\hline$\# 4$ & Staggered & 24 \\
\hline$\# 5$ & In-line & 8 \\
\hline$\# 6$ & In-line & 12 \\
\hline$\# 7$ & In-line & 16 \\
\hline$\# 8$ & In-line & 20 \\
\hline$\# 9$ & In-line & 24 \\
\hline
\end{tabular}

\section{RESULTS}

The analysis of the results is divided in two contributions at first: pressure losses and heat transfer analyses; secondly, a comparison of the results with optimal diameter analysis and overall performance coefficient is discussed.

\subsection{Pressure drops}

The first stage concerns the study of overall pressure drops in both the tubes and the shell.

Figure 4 and 5 show the trends of pressure drops generated, respectively in the shell-side and in the tube-side, with increasing inner diameter.

In order to clarify how the diameter growth affects the two arrangements (aligned and staggered), in

Figure 4 and Figure 5 pressure drops are represented with a logarithmic scale. The graphs show, respectively, the pressure drop that occur internally both in the cold and hot side for the 10 cases.

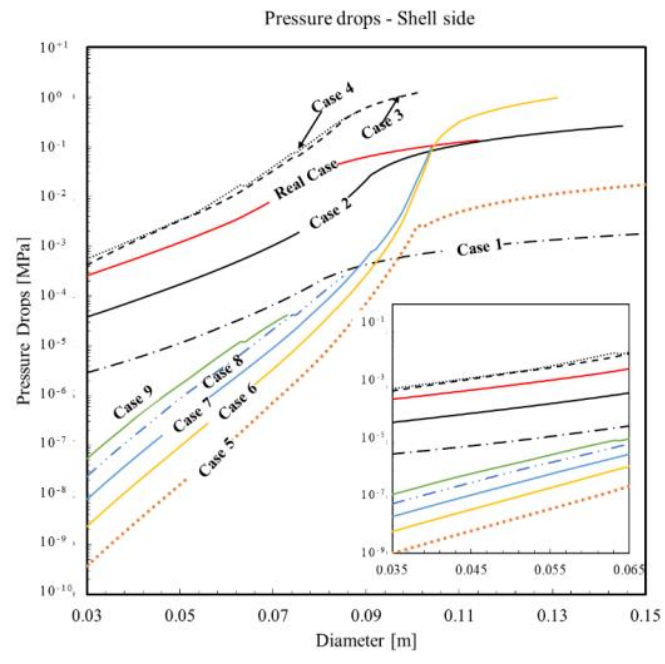

Figure 4. Pressure drops - shell side 
Thanks to the smaller number of deviations to which the fluid is subject, the cross-flow configuration with the aligned tubes generates lower pressure drops in the shell side compared to those generated by the heat exchanger with staggered tubes. For example, with equal numbers of tubes, the real case generates pressure drops greater than $2 \times 10^{-3}$ $\mathrm{MPa}$ if compared to Case 7 ; then, when the diameter is approximately $100 \mathrm{~mm}$, the curves are very close. The highest values are traced for Case 3 and 4 that are only to a limited extent higher than the real case. The lowest results are reported for case 5 (aligned with 8 tubes).

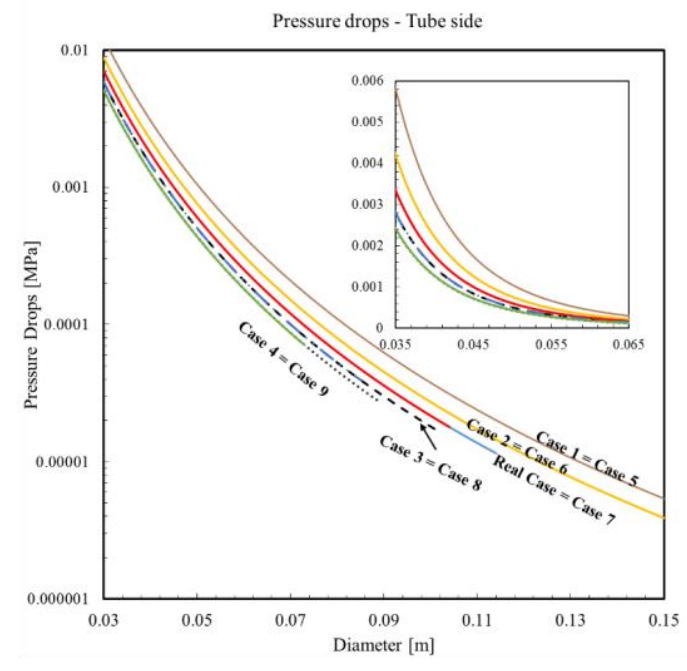

Figure 5. Pressure drop - flow inside tube

Figure 5 describes instead the pressure drops that are generated in the tubes while increasing their number, from 8 in Case 1 and 5 to Case 4 and 9 with 24 tubes. Generally, pressure drops decrease with the increase of the number of pipes. The results depend strongly on the number of tubes; the trends of pressure drops of the aligned cases are comparable to those of the staggered cases.

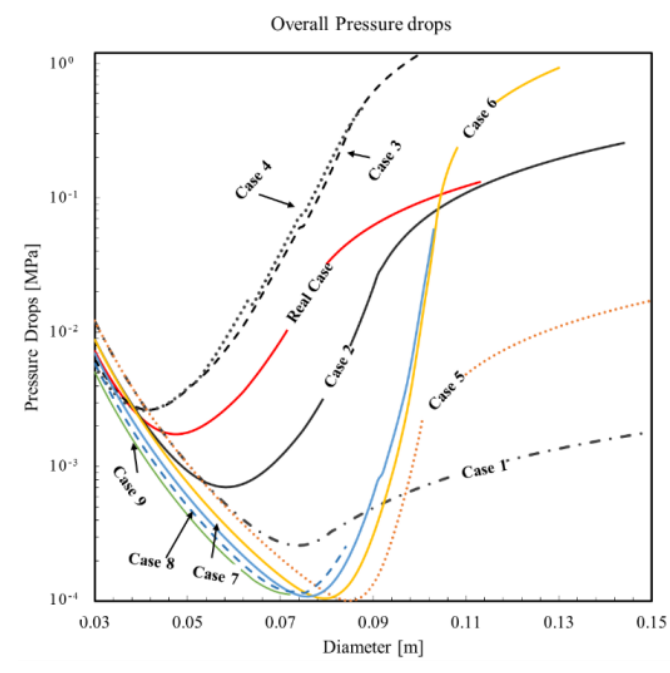

Figure 6. Overall pressure drops

Figure 6 shows the trends of overall pressure drops.
Overall pressure drops (Eq. 19) is the total of the pressure drops generated inside and outside the bank of tubes.

$\Delta P_{\text {overall }}=\Delta P_{\text {cold }}+\Delta P_{\text {hot }}$

The real case presents the global minimum of overall pressure drops close to the actual diameter of $50 \mathrm{~mm}$. In cases 3 and 4, however, the lower value is for diameter values close to $30-40 \mathrm{~mm}$. Other cases have the same trend; but they have the minimum in the vicinity of larger diameters.

\subsection{Thermal analysis}

The second analysis concerns the study of the heat exchanged by the boiler as function of the tubes' diameter.

From the point of view of the sizing of the boiler, it is necessary that the machine is able to meet the required thermal load. For this reason, Figure 7 shows heat power as function of the diameter of the tubes.

Typically, the convection coefficient of a row increases with increasing row number, after which there is little change in flow conditions and hence in the convection coefficient. For large $S_{L}$, the influence of upstream rows decreases, and heat transfer in the downstream rows is not enhanced. For this reason, operation of aligned tube banks with $\mathrm{S}_{\mathrm{T}} / \mathrm{S}_{\mathrm{L}}<0.7$ is undesirable [18].

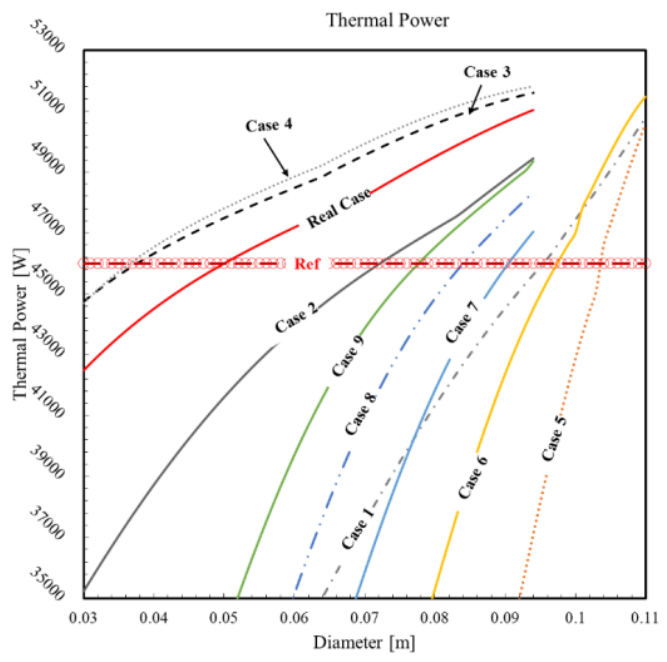

Figure 7. Thermal Power transfer

The dotted line called "Ref" shows the thermal power required by the user $(46 \mathrm{~kW})$. Its representation is needed to understand if and where the trend of thermal power developed in each case reaches the required thermal power

As can be observed from Figure 7, the trend of the thermal power for the various cases, intersects the reference line for different diameters. The case, requiring the least diameter to cover the thermal load, is the case 4 . Case 9 , also, required a diameter equal to $78 \mathrm{~mm}$, but the optimum diameter exceeds the threshold diameter by failing to ensure the normal course of the cold fluid. 
Table 4. Summary of the optimal data for the analyzed configurations

\begin{tabular}{lccccccccccc}
\hline & & Real Case & Case \#1 & Case \#2 & Case \#3 & Case \#4 & Case \#5 & Case \#6 & Case \#7 & Case \#8 & Case \#9 \\
\hline $\mathrm{di}$ & {$[\mathrm{mm}]$} & 50 & 96 & 73 & 38 & 37 & 104 & 98 & 91 & 84 & 78 \\
\hline $\mathrm{do}$ & {$[\mathrm{mm}]$} & 55 & 101 & 78 & 43 & 42 & 109 & 103 & 96 & 89 & 83 \\
\hline Number of tubes & - & 16 & 8 & 12 & 20 & 24 & 8 & 12 & 16 & 20 & 24 \\
\hline Pressure Drop shell side & $10^{-5}[\mathrm{MPa}]$ & 119.70 & 302.48 & 143.14 & 119.75 & 90.26 & 1256.49 & 352.53 & 76.11 & 21.09 & 6.71 \\
\hline Pressure Drop tube-side & $10^{-5}[\mathrm{MPa}]$ & 60.33 & 4.59 & 12.35 & 188.40 & 185.06 & 3.12 & 3.00 & 3.41 & 4.18 & 5.16 \\
\hline Overall pressure drop & $10^{-5}[\mathrm{MPa}]$ & 180.02 & 307.07 & 155.49 & 308.15 & 275.33 & 1259.61 & 355.53 & 79.52 & 25.27 & 11.87 \\
\hline Thermal Power & {$[\mathrm{kW}]$} & 46.00 & 46.16 & 46.13 & 46.06 & 46.08 & 46.55 & 46.24 & 46.17 & 46.01 & 46.08 \\
\hline Epsilon & $\%$ & 88.53 & 88.84 & 88.77 & 88.65 & 88.68 & 89.59 & 89.00 & 88.85 & 88.55 & 88.68 \\
\hline Exchange surface & {$\left[\mathrm{m}^{2}\right]$} & 1.66 & 1.52 & 1.76 & 1.62 & 1.90 & 1.64 & 2.33 & 2.90 & 3.36 & 3.75 \\
\hline
\end{tabular}

\subsection{Optimal inner diameter}

The optimal diameter was calculated for each configuration on the basis of the required heat load $(46 \mathrm{~kW})$.

These graphs show that although case 5 and case 6 are characterized by small pressure drops both in the shell side and in the tube side, they are able to cover the thermal user requirements only with diameters larger than $90 \mathrm{~mm}$. This would cause very large overall pressure drops as in Figure 6.

If the diameter of the tubes are considered constant, the resulting pressure drops and thermal power are reported in Table 3.For the 10 cases analyzed it was determined that the optimal diameter allows to obtain the pressure drops and the thermal power required as shown in Table 4.

The geometry, the number and arrangements of longitudinal pipes affect both the pressure drops and the amount of heat exchanged in the boiler.

Table 3. Results for constant inner diameter (50 mm)

\begin{tabular}{llll}
\hline Case & $\begin{array}{l}\mathrm{d}_{\mathrm{i}} \\
{[\mathrm{m}]}\end{array}$ & $\begin{array}{l}\text { Overall pressure drop } \\
10^{-5}[\mathrm{MPa}]\end{array}$ & $\begin{array}{l}\text { Thermal Power } \\
{[\mathrm{kW}]}\end{array}$ \\
\hline Real Case & 0.05 & 180.02 & 46.00 \\
\hline Case \#1 & 0.05 & 106.17 & 29.11 \\
\hline Case \#2 & 0.05 & 93.12 & 41.61 \\
\hline Case \#3 & 0.05 & 381.24 & 47.50 \\
\hline Case \#4 & 0.05 & 372.55 & 47.81 \\
\hline Case \#5 & 0.05 & 105.04 & 9.87 \\
\hline Case \#6 & 0.05 & 75.95 & 16.24 \\
\hline Case \#7 & 0.05 & 60.36 & 22.78 \\
\hline Case \#8 & 0.05 & 50.55 & 28.37 \\
\hline Case \#9 & 0.05 & 43.78 & 33.75 \\
\hline
\end{tabular}

The optimal diameter for cases 3 and 4, is equal to 38 and $37 \mathrm{~mm}$ respectively, but the pressure drops which are generated are higher than those that are generated in the real case. Increasing the internal diameter would allow for further nominal heat output of the machine and consequently lower drops of tube side pressure.

In any case, the actual boiler has pressure drops in line with the average of the cases.

The best cases are the 2, 7, 8 and 9 which have pressure drops respectively equal $14 \%, 54 \%, 86 \%$ and $93 \%$ less than real case.

\subsection{Overall performance coefficient application}

In [16] a concept of performance analysis determined by the contribution of the pressure loss and heat removed, applied to fins in heat exchanger, was developed

In this work, the Overall Performance Coefficient was applied to quantify the relative relevance of pressure drops and heat exchanged in the parametric analysis proposed.

The overall performance coefficient is calculated as in Eq.19:

$P_{1}^{*}=\alpha \tilde{Q}_{i}+(1-\alpha) \frac{1}{\Delta P_{i}^{*}}$

where $\mathrm{Q}_{\mathrm{i}}$ is the dimensionless heat transfer in the $\mathrm{i}_{\text {th }}$ case, calculated as ratio between heat transfer in the $\mathrm{i}_{\text {th }}$ case and heat transfer in the real case [16]; while $\Delta \mathrm{P}^{*}{ }_{\mathrm{i}}$ is a mean dimensionless relative loss of pressure in the $\mathrm{i}_{\text {th }}$ case respect real case and $\alpha$ is called relevance, varying between 0 and 1 , representing the weight of heat transfer maximization with respect to loss of pressure minimization, depending on the context and on the use of the heat exchanger. The results are shown in Figure 8.

Because of the pressure drop detected in the cases 7,8 and 9 smaller than case of reference, it has opted for a graphical representation that does not show these trends for entire in Figure 8, thus obtaining a clearer understanding of the results.

The results report that, for optimal diameter, the trend of cases is not constant. The Table 4 shows how the arrangement of the pipes inside the heat exchanger results in the variation of the pressure drops and the efficiency of the boiler. If $\alpha$ is 0 , the overall performance coefficient, for the case 7 , results 2.26 , for the case 8 and case 9 are, respectively, 7.12 and 15.17; others are approximately equal to the reference case. If in the first part of the graph, the curves differ from each other, instead if $\alpha$ is 1 , the trends converge towards the real case, in fact, the first prerequisite of the paper is the respect of the thermal power by varying the geometry. All curves converge on the right side for $\alpha=1$ on the same point, that corresponds to the required thermal power of the machine, while the best performing solution would seem Case 9 (Aligned with 24 tubes), or in other words the scenario with the lowest pressure drops.

In fact, the best case is the minor overall pressure drops to cover thermal power required. 


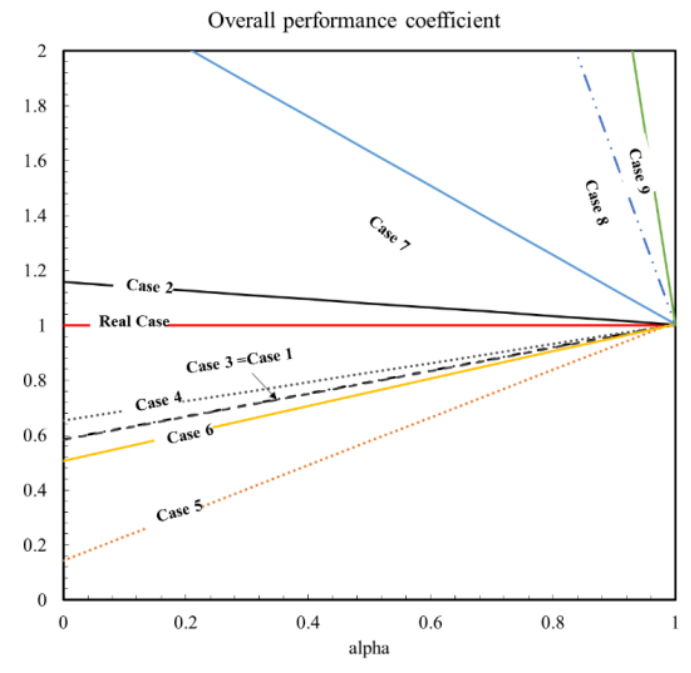

Figure 8. Overall performance coefficient in function of heat transfer

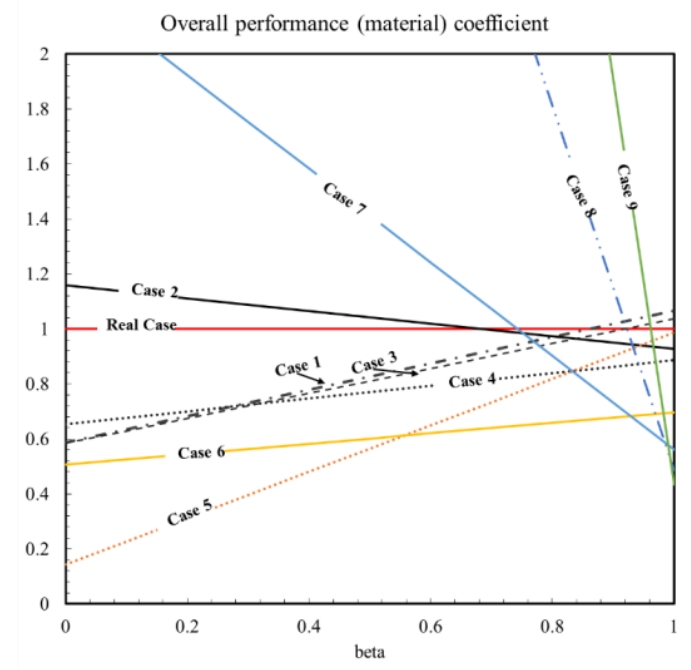

Figure 9. Overall performance (material) coefficient in function of volume of tubes

However, since the base assumption of the analysis is that the prerequisite of the pellet burner is to be able to cover and guarantee $46 \mathrm{~kW}$ of heat power, it makes sense to also reinterpret the Overall performance coefficient and to better adapt it to the context. In fact, if the heat power achievable by the boiler is fixed and the idea of reducing the amount of materials - and indirectly the amount of energy used to produce them - needed to achieve the same results is introduced, it is possible to state that Overall performance coefficient in the second scenario can be calculated as in the following Eq.20:

$$
P_{2}^{*}=\beta \frac{1}{\tilde{V}_{i}}+(1-\beta) \frac{1}{\Delta P_{i}}
$$

where $\mathrm{V}_{\mathrm{i}}$, is the dimensionless volume of tubes in the $\mathrm{i}_{\text {th }}$ case, calculated as ratio between volume of tubes in the $i_{\text {th }}$ case and volume of tubes in the real case [16] and $\beta$ can be defined relevance of materials, varying between 0 and 1 , representing the weight of volume of materials minimization with respect to loss of pressure minimization.

In particular, if $\beta$ is set equal to 1 this means that, for the technical use requested, $\mathrm{P}_{2}{ }^{*}$ is equal to the inverse ratio of dimensionless volume, if $\beta$ is set equal to 0 this means that $\mathrm{P}_{2}{ }^{*}$ is equal to the dimensionless inverse ratio of pressure drops. Each value of relevance between 0 and 1 will describe intermediate needs.

It is desirable under these assumptions, that $\mathrm{P}_{2}{ }^{*}$ is as high as possible.

Results mark in this case, a heterogeneous scenario with diverging results. There appears not to be a best option from all points of view but the optimal solution needs to be identified through trade-offs. In particular there isn't a better global case respect to the real case, in fact minor pressure drops are connected to major volume of material of tubes. From this point of view it appears that the real case could be considered a good tradeoff between material use and pressure drops. In fact, Case 2 has values close to those of the real case in terms of both volume and pressure drops, presenting lower pressure drop and greater quantities of material used, instead Cases 1 and 3 have reversed trends. Cases 4, 5 and 6, have higher pressure drops and volume of materials employed.

\section{CONCLUSIONS}

The paper reports a parametric analysis under multiple points of view considering the Costructal Law as main inspiration for the design variation.

The parametric analysis considers the variation of most geometrical parameters of the boiler, including the diameter size, the positioning and the numbering of the tubes, while keeping the boiler volume constrained to the existing machine.

Results are presented in terms of heat power variation and overall pressure drops in both the shell and tubes sides. The application of the Overall performance coefficient leads to a comprehensive perspective on the results, identifying that the best performing solution is the aligned configuration with 24 tubes; but if volume of materials of tubes is introduced as variable, the results are more heterogeneous, because there isn't an overall best case if compared to the real case and the best solution can be identified only through trade-offs.

The analysis proposed in the paper marks one of the first applications to boilers of Constructal law and introduces the material uses perspective as a parameter to achieve a design optimization of the system under examination.

It is particularly needed to take care in the selection of relevance used in the overall performance coefficient method since the results are heavily influenced by it.

Moreover, the paper marks the introduction of an ecodesign inspired optimization in the Overall performance coefficient calculation as one of the two optimization pillars in the methodology.

\section{REFERENCES}

[1] Manjunath K., Kaushik S.C. (2014). Second law thermodynamic study of heat exchangers: a review, Renewable and Sustainable Energy Reviews, Vol. 40, pp. 348-374.

[2] Bejan A. (1997). Constructal-theory network of conducting paths for cooling a heat generating volume, International Journal of Heat and Mass Transfer, Vol. 
40, pp. 799-816.

[3] Bejan A., Lorente S. (2004). The constructal law and the thermodynamics of flow systems with configuration, International Journal of Heat and Mass Transfer, Vol. 47, pp. 3203-3214.

[4] Chen L., Feng H., Xie Z., Sun F. (2015). Thermal efficiency maximization for $\mathrm{H}$ - and $\mathrm{X}$-shaped heat exchangers based on constructal theory, Applied Thermal Engineering, Vol. 91, pp. 456-462.

[5] Yang J., Fan A., Liu W., Jacobi A.M. (2014). Optimization of shell-and-tube heat exchangers conforming to TEMA standards with designs motivated by constructal theory, Energy Conversion and Management, Vol. 78, pp. 468-476.

[6] Manjunath K., Kaushik S.C. (2014). The second law analysis of an unbalanced constructal heat exchanger, International Journal of Green Energy, Vol. 11, pp. 173-192.

[7] Manjunath K., Kaushik S.C. (2014). Entropy generation and thermo-economic analysis of constructal heat exchanger, Heat Transfer - Asian Research, Vol. 43, pp. 39-60.

[8] Zimparov V.D., da Silva A.K., Bejan A. (2006). Constructal tree-shaped parallel flow heat exchangers, International Journal of Heat and Mass Transfer, Vol. 49, pp. 4558-4566.

[9] Da Silva A.K., Bejan A. (2006). Dendritic counterflow heat exchanger experiments, International Journal of Thermal Sciences, Vol. 45, pp. 860-869.

[10] Da Silva A.K., Lorente S., Bejan A. (2004). Constructal multi-scale tree-shaped heat exchangers, Journal of Applied Physics, Vol. 96, pp. 1709-1718.

[11] Lorenzini G., Rocha L.A.O. (2009). Geometric optimization of T-Y-shaped cavity according to Constructal design, International Journal of Heat and Mass Transfer, Vol. 52, pp. 4683-4688.

[12] A. Bejan (2002). Dendritic constructal heat exchanger with small-scale crossflows and larger-scales counterflows, International Journal of Heat and Mass Transfer, Vol. 45, pp. 4607-4620.

[13] Liu X., Chen L., Feng H., Qin X., Sun F. (2016). Constructal design of a blast furnace iron-making process based on multi-objective optimization, Energy, Vol. 109, pp. 137-151.

[14] Kang D.H., Lorente S., Bejan A. (2010). Constructal architecture for heating a stream by convection, International Journal of Heat and Mass Transfer, Vol. 53, pp. 2248-2255.

[15] Koonsrisuk A., Lorente S., Bejan A. (2010). Constructal solar chimney configuration, International Journal of Heat and Mass Transfer, Vol. 53, pp. 327333.

[16] Lorenzini G., Moretti S. (2009). A. Bejan's constructal theory approach to the overall optimization of heat exchanging finned modules with air in forced convection and laminar flow condition, Journal of Heat Transfer, Vol. 131, p. 081801.

[17] Khan W.A., Culham J.R., Yovanovich M.M. (2006). Convection heat transfer from tube banks in crossflow: Analytical approach, International Journal of Heat and Mass Transfer, Vol. 49, pp. 4831-4838.

[18] Incropera F.P., DeWitt D., Bergman T. (2011). Fundamentals of heat and mass transfer, J.W. Sons, Ed., seventh ed., pp. 468-476,517-593.
[19] Žukauskas A., Ulinskas R. (1985). Efficiency parameters for heat transfer in tube banks, Heat Transfer Engineering, Vol. 6, pp. 19-25.

\section{NOMENCLATURE}

A Heat transfer surface $\left(\mathrm{m}^{2}\right)$

C Correction factor for Nusselt number

$\mathrm{c}_{\mathrm{p}} \quad$ Specific heat capacity at constant pressure $\left(\mathrm{J} \mathrm{kg}^{-1}\right.$

$\left.\mathrm{c}_{\mathrm{p}} \quad \mathrm{k}^{-1}\right)$

d Diameter $(\mathrm{m})$

F LMTD correction factor

$f \quad$ Friction coefficient (-)

$\mathrm{h}$ Convective heat transfer coefficient for one fluid

(W m $\left.{ }^{-2} \mathrm{~K}^{-1}\right)$

$\mathrm{H} \quad$ Height

$\mathrm{k} \quad$ Thermal conductivity $\left(\mathrm{W} \mathrm{m}^{-1} \mathrm{~K}^{-1}\right.$ )

$\mathrm{L} \quad$ Length of the heat exchanger channel (m)

M Mass flow rate $\left(\mathrm{kg} \mathrm{s}^{-1}\right)$

$\mathrm{N} \quad$ Number of the tubes

$\mathrm{Nu} \quad$ Nusselt number

$\mathrm{P}^{*} \quad$ Overall performance coefficient function of heat

P transfer

$\mathrm{P}_{2}^{*} \quad$ Overall performance coefficient function of

$\mathrm{P}_{\mathrm{L}} \quad$ Dimensionless longitudinal pintch

Pr Prandtl number

$\mathrm{P}_{\mathrm{T}} \quad$ Dimensionless trasfersal pintch

Q Heat flow $(\mathrm{kW})$

$\mathrm{R}_{\mathrm{f}} \quad$ Fouling factor $\left(\mathrm{m}^{2} \mathrm{~K} / \mathrm{W}\right)$

Re Reynolds number

$\mathrm{S}_{\mathrm{D}} \quad$ Diagonal pintch $(\mathrm{m})$

$\mathrm{S}_{\mathrm{L}} \quad$ Longitudinal pintch $(\mathrm{m})$

$\mathrm{S}_{\mathrm{L}} \quad$ Trasfersal pintch $(\mathrm{m})$

$\mathrm{T} \quad$ Temperature $(\mathrm{K})$

$\mathrm{U} \quad$ Overall heat transfer coefficient $\left(\mathrm{W} \mathrm{m} \mathrm{m}^{-2} \mathrm{~K}^{-1}\right)$

$\mathrm{u} \quad$ Velocity of flow $\left(\mathrm{m} \mathrm{s}^{-1}\right)$

W Width (m)

$\Delta \mathrm{P} \quad$ Pressure drop (MPa)

$\Delta \mathrm{P}_{\text {overall }}$ Overall pressure drop $(\mathrm{MPa})$

$\Delta \mathrm{T}_{\mathrm{lm}} \quad$ Mean log-temperature difference $(\mathrm{K})$

\section{Greek symbols}

$\begin{array}{ll}\alpha & \text { Relative } \\ \beta & \text { Relative of materials } \\ \mu & \text { Viscosity }\left(\mathrm{kg} \mathrm{m}^{-1} \mathrm{~s}^{-1}\right) \\ \mu_{\mathrm{w}} & \text { Viscosity in wall temperature }\left(\mathrm{kg} \mathrm{m}^{-1} \mathrm{~s}^{-1}\right) \\ \rho & \text { Density of the fluid }\left(\mathrm{kg} \mathrm{m}^{-3}\right)\end{array}$

\section{Subscripts}

cold Cold fluid 
hot Hot fluid

In

$\max \quad$ Max value

o Out p Conventional pyramid distributor

r Rows

s Shell-side

t Tube-side 